University of Wollongong

Research Online

Faculty of Engineering and Information

Faculty of Engineering and Information

Sciences - Papers: Part A

Sciences

$1-2-2014$

Ion radiography as a tool for patient set-up \& image guided particle therapy: a Monte Carlo study

Nicolas Depauw

University of Wollongong, nd999@uowmail.edu.au

Marta F. Dias

University of Lisbon

Anatoly B. Rosenfeld

University of Wollongong, anatoly@uow.edu.au

Joao C. Seco

Harvard Medical School, Boston, jseco@partners.org

Follow this and additional works at: https://ro.uow.edu.au/eispapers

Part of the Engineering Commons, and the Science and Technology Studies Commons

Research Online is the open access institutional repository for the University of Wollongong. For further information contact the UOW Library: research-pubs@uow.edu.au 


\title{
Ion radiography as a tool for patient set-up \& image guided particle therapy: a Monte Carlo study
}

\author{
Abstract \\ This study investigate the use of ion radiography as a tool for patient set-up and tumor tracking \\ capabilities for image guided particle therapy (IGPT) using Monte Carlo simulations. One pediatric, two \\ lung and one liver cancer patients were considered in this study. For each patient, 230 and $330 \mathrm{MeV}$ \\ proton, and $500 \mathrm{MeV} /$ nucleon carbon ion pencil beams were simulated through their computed \\ tomography (CT) data set using GEANT4.9.0. Energy, position and direction cosines of each particle were \\ recorded in front and behind the patient. Ion radiographs were subsequently reconstructed using a \\ dedicated in-house software. The image quality was assessed by evaluating the contrast-to-noise ratio of \\ the tumor and its surrounding tissue. In the lung and liver cases, each CT phase of the breathing cycle \\ was treated individually and dynamic sequences were later produced to appreciate tumor motion. \\ Reconstructed radiographs show high spatial resolution. This allows for excellent imaging capabilities in \\ pediatric patients, comparable to X-ray imaging at a fraction of the imaging dose. There is clear \\ visualization of the tumor edges in the lung due to the great contrast-to-noise ratio between the tumor \\ and its surrounding tissues; tumor motion is observed and comparable to 4D CT data thus allowing for \\ on-line tumor tracking during ion radiotherapy. Conversely, tumor edge detection is difficult in liver, and \\ fiducial markers are required to attempt indirect tumor tracking for IGPT. Ion radiographs with high spatial \\ resolution can be generated using the PR-creator software resulting in pediatric patient set-up capabilities \\ at a fraction of the current imaging dose, as well as the capacity to track moving targets in order to \\ achieve IGPT.
}

\section{Keywords}

patient, setup, monte, carlo, ion, study, radiography, tool, guided, particle, therapy, image, 76, 69

\section{Disciplines}

Engineering | Science and Technology Studies

\section{Publication Details}

Depauw, N., Dias, M. F., Rosenfeld, A. \& Seco, J. C. (2014). Ion radiography as a tool for patient set-up \& image guided particle therapy: a Monte Carlo study. Technology in Cancer Research and Treatment, 13 (1), 69-76. 


\title{
Ion radiography as a tool for patient setup \& IGIT: a Monte Carlo study.
}

\author{
Nicolas Depauw ${ }^{1,2}$, Marta F. Dias ${ }^{1,3}$, Anatoly Rosenfeld ${ }^{2}$, Joao Seco ${ }^{1}$ \\ ${ }^{1}$ Francis H. Burr Proton Therapy Center, Department of Radiation Oncology, \\ Massachusetts General Hospital (MGH), Boston, MA, USA \\ ${ }^{2}$ Center for Medical Radiation Physics (CMRP), University of Wollongong (UoW), NSW, Australia \\ ${ }^{3}$ Institute of Biophysics and Biomedical Engineering, Faculty of Sciences of the University of Lisbon, Portugal
}

\begin{abstract}
Purpose: To investigate the use of ion radiography as a tool for patient setup and tumor tracking capabilities for image guided ion therapy (IGIT) using Monte Carlo simulations.

Methods: One pediatric, two lung and one liver cancer patients were considered in this study. For each patient, 230and 330- $\mathrm{MeV}$ proton, and 500- $\mathrm{MeV} /$ nucleon carbon ion pencil beams were simulated through their CT data set using GEANT4.9.0. Energy, position and direction cosines of each particle were recorded in front and behind the patient. Ion radiographs were subsequently reconstructed using the PR-creator software. In the lung and liver cases, each CT phases of the breathing cycle was treated individually, and dynamic sequences were later produced to appreciate tumor motion.

Results: Reconstructed radiographs show high spatial resolution $(\approx 1.2-1.6 \mathrm{~mm})$. This allows for excellent imaging capabilities in pediatric patients, comparable to X-ray imaging at a fraction of the imaging dose. There is clear visualization of the tumor edges in lung due to the great contrast-to-noise ratio between the tumor and its surrounding tissues; tumor motion is observed and comparable to $4 \mathrm{D} \mathrm{CT}$ data $(\approx 0.5-1 \mathrm{~cm})$, thus allowing for on-line tumor tracking during ion radiotherapy. Conversely, tumor edge detection is difficult in liver, and fiducial markers are required to attempt indirect tumor tracking for IGIT.

Conclusions: Ion radiographs with high spatial resolution can be generated using the PR-creator software resulting in pediatric patient setup capabilities at a fraction of the current imaging dose, as well as the capacity to track moving targets in order to achieve IGIT.
\end{abstract}

\section{${ }_{20} \quad 1$ Introduction}

Proton radiography, as a radiological tool, was first investigated in the early 1970s and 1980s [1]-[2]. However, there was not a real need for such technology at the time, and today's conventional x-ray radiography quickly became the most common cancer diagnostic tool in hospitals. Protons, and more generally ions, have nonetheless been increasingly attractive to the radiotherapy community due to their finite dose distribution. Proton and carbon ion radiotherapy centers are slowly proliferating, and more appropriate tools are needed in order to fully take advantage of ion's intrinsic dosimetric qualities.

Currently, clinics relay on x-ray imaging for patient setup and external tools for tumor tracking in order to achieve image guided radiation therapy. The use of ion radiography could therefore represent a significant improvement in ion radiotherapy: from a patient set-up point of view, this would reduce the potential misalignment between the set-up x-ray beam's eye view and the therapeutic ion beam's eye view while greatly reducing the imaging dose delivered to a given patient; from an Image Guided Ion Therapy (IGIT) standpoint, it could be used seamlessly during treatment for tumor tracking and on-line dose delivery adjustment. 
Conventional x-ray radiographs are simple fluence images behind the patient, on the other hand, ion radiographs are slightly more complicated to produce as they correspond to "range maps" [3] of the patient. Currently, the main limitation of ion therapy resides in the range uncertainties due to cavities and other morphological changes that occur over the duration of the treatment. Thus, ion radiography, by providing water-equivalent thickness (WET) data through the patient, could further help reduce these range uncertainties in the clinic practice.

Recent work on proton radiography has been taking a special interest in its intrinsic imaging qualities [3]-[8]. Although proton radiography shows poor spatial resolution compare to x-ray radiography, it shows much better density resolution [8] which is of great importance for defining tumor edges and help tracking a moving target. The physical properties of carbon ions are very similar to the ones of protons, and all results are expendable. However, carbon ions do not suffer Multiple Coulomb Scattering (MCS) which would therefore result in a significantly higher spatial resolution.

This study focuses on the feasibility to use ion imaging, specifically radiography, as a tool for daily patient set-up and image guided therapy through on-line tumor tracking. Different categories of patients were selected in order to show distinct advantages. Due to the novelty of the subject, this study is entirely based on computer simulations in order to suppress the bias from the current state of the art in ion detector technology[9].

\section{Materials and methods}

\subsection{Patients}

Three types of cancer patients were selected for this study: one pediatric, two lung, and one liver cases.

Radiation doses to pediatric patients are extremely relevant as it can results in growth issues and later risk of malignancies. Consequently, imaging doses should be minimized. The pediatric case consists of a baby's' full body scan. It is a relevant case to investigate the ratio quality/dose in comparison with conventional X-ray imaging.

In order to look at direct tumor tracking using ion radiography, two lung tumor patients were selected. The first case is a female patient presenting a tumor in the lower region of the right lung with a significant motion amplitude $(\approx 1 \mathrm{~cm})$. The $(\approx 0.5 \mathrm{~cm})$.

Finally, a liver case was selected due to the presence of fiducial markers. The latter is relevant to investigate the feasibility of using ion radiography for indirect tumor tracking.

\subsection{Monte Carlo model} described by Jarlskog and Paganetti[11] were used in order to correctly track ions. Scanned pencil beams (monochromatic and unidirectional) of 230- and 330- $\mathrm{MeV}$ protons, as well as 500- $\mathrm{MeV} /$ nucleon carbon ions, covering a 40-cm square homogeneous area, were simulated through the patients. On average, twenty million histories (particles) were simulated to produce proton and carbon ion images.

For each radiograph production, the corresponding patient computed tomography (CT) data was imported into GEANT4. The Hounsfield unit in each voxel was subsequently converted to a corresponding material definition; this conversion, improved from Paganetti et al's work [12], includes 24 different tissue definitions with a total of 4,071 densities, and therefore allows for proper interaction calculations.

Energy, Cartesian coordinates and direction cosines were recorded for each primary particle crossing a plane $1 \mathrm{~cm}$ before the patient, as well as for each primary or secondary ions crossing a plane $1 \mathrm{~cm}$ behind the subject. Furthermore, a trackID number was registered for each event in order to appropriately associate a particle in the rear plane to its incident particle in the fore plane. The setup is shown in figure 1. 


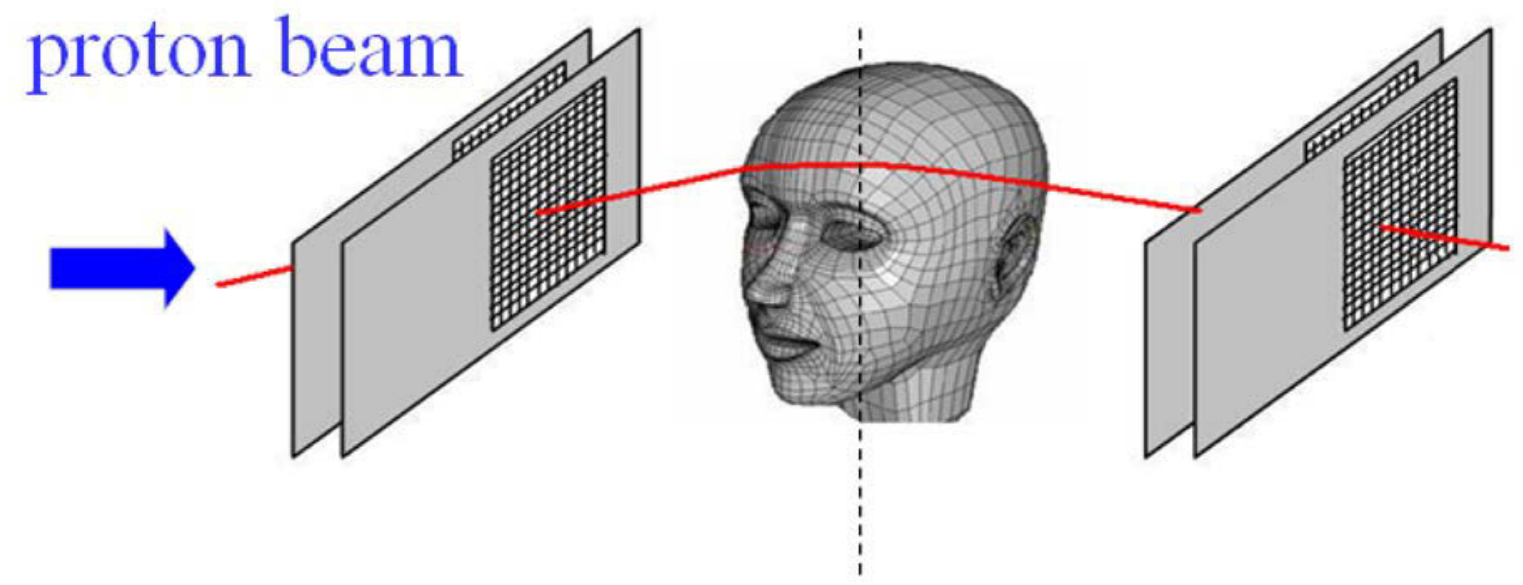

Figure 1: Set-up for simulating ion radiographs through a patient CT using the GEANT4 Monte Carlo toolkit.

\subsection{Image reconstruction}

75 Ion radiographs are range maps; each pixel value represents the range of the absorbed energy $R_{a b s}$. The latter corresponds to the range in water of the mean energy loss of all the ions going through that given pixel. The reconstruction algorithm, based on a "most likely path" technique [3], is not described in details here. An ion radiographs reconstruction software,

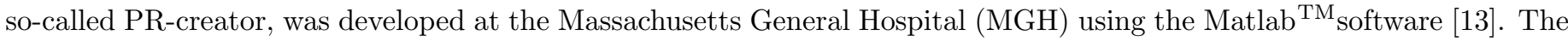
PR-creator allows the user to load the data from Monte Carlo simulations (the code can easily be modified to consider experimental data) and reconstructs ion radiographs based on different options (see Figure 2). In this study, radiographs were generated as $1024 \times 1024$ pixel images, corresponding to a $0.4 \mathrm{~mm} /$ pixel resolution, along with appropriately chosen energetic and angular cuts to fully appreciate the tumor location. It should be noted that the software gives the possibility to a physician or therapist to apply a windowing filter on a specific region of interest (ROI) which can further help locating the tumor edges.

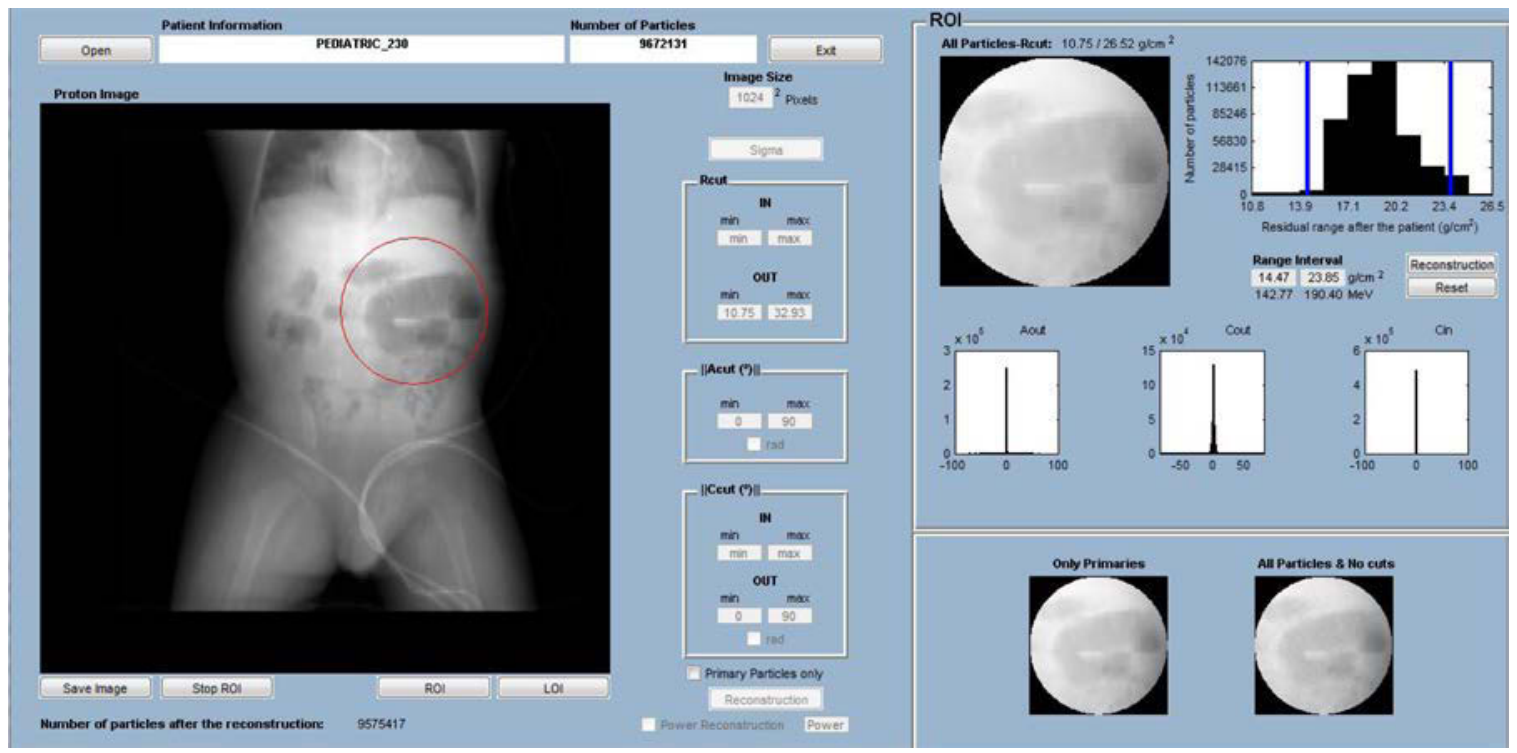

Figure 2: PR-creator software developed at MGH. The user can chose to reconstruct radiographs taking into account only primary ions or both primary and secondary ions, and apply energetic (range), angular and directional cuts. In a real case scenario, applying the appropriate cuts would significantly improve the output image quality [8]. The software also includes a region-of-interest (ROI) option that allows the user to further analyze a specific region in the image.

When relevant, and in order to fully appreciate the tumor motion, breathing cycle sequences were produced using $\mathrm{IAT}_{\mathrm{E}} \mathrm{X}$; 
each CT phase radiograph was individually reconstructed beforehand using the PR-creator software.

\section{Results and discussion}

\subsection{Pediatric patient}

Figure 3 shows the original CT data set and a generated proton radiograph side-by-side for the pediatric patient.
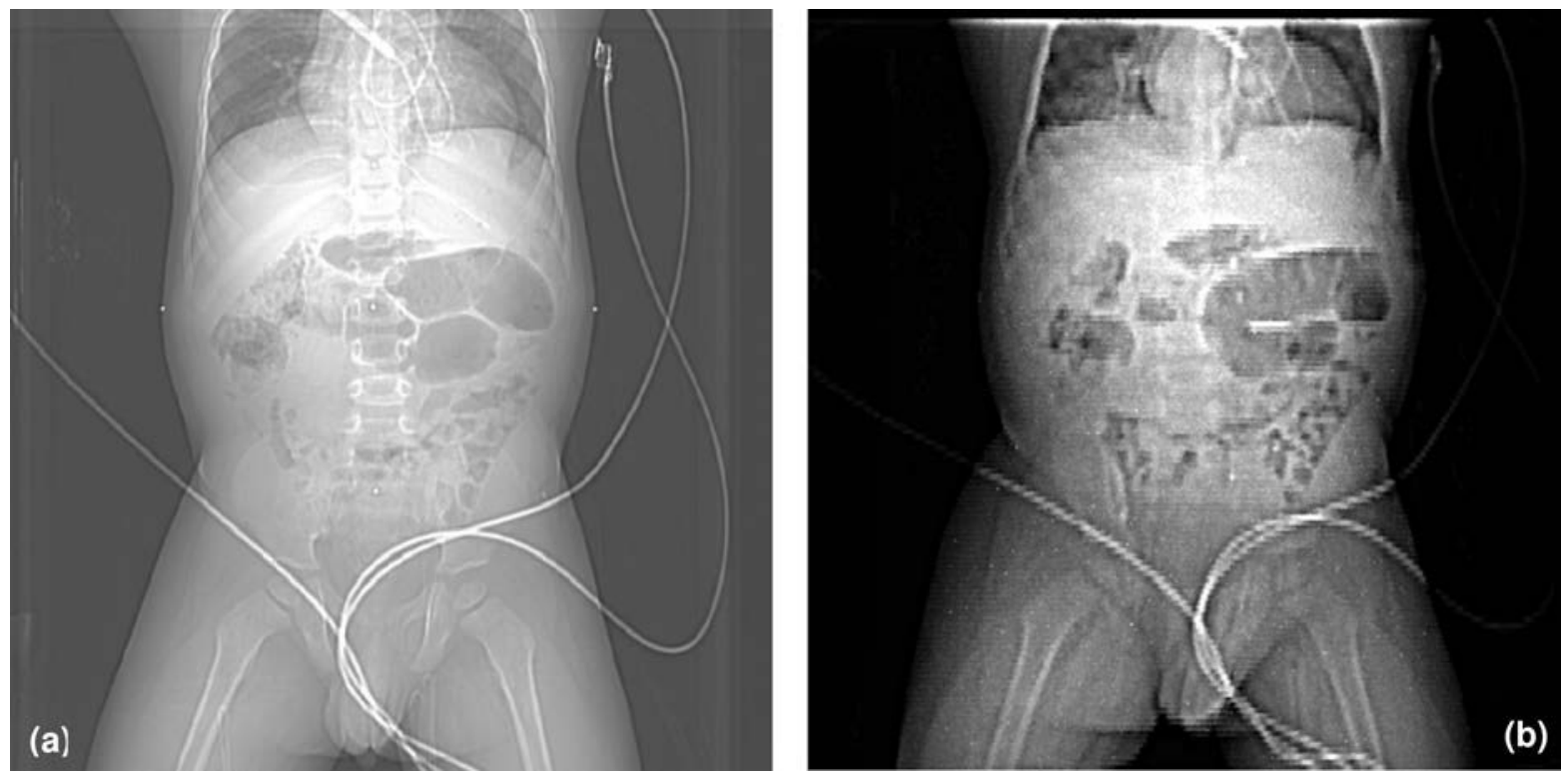

Figure 3: a) Original $C T$ and b)reconstructed 230-MeV proton radiograph of a pediatric patient's full body scan.

known limitation of proton radiography resides in its poor intrinsic spatial resolution [3]. However, it was shown in a previous study, performed with a less efficient version of the PR-creator software, that applying the appropriate cuts in range (energy) and angular distribution results in clinically relevant image's quality [8]. The reported resolution has been further improved and more work is in progress in order to obtain, in the best cases, sub-millimeter spatial resolution. It should be noted that, in the patient simulations, there are small uncertainties in the Hounsfield unit values of the CT $(\approx 1 \%)$, and, possibly, in the material conversion in GEANT4. This could therefore be responsible for a slight decrease of the best achievable spatial resolution.

In such pediatric cases, this all translates to a high imaging quality, comparable to a conventional X-ray. The bony structures are clearly visible which therefore allows for patient setup prior to radiotherapy. Based on our recent study, the twenty millions particles used to produce this proton radiograph accounts for approximately $0.002 \mathrm{mSv}[8]$. On the other hand, the Health Physics Society recommends a delivered dose of approximately $0.02 \mathrm{mSv}$ for a current conventional chest X-ray[14]-[15]. This clearly emphasizes the possible gain in using proton radiography for patient setup purposes as the imaging dose could be reduced by an order of magnitude. This is particularly relevant in pediatric patient for which small radiation dose often leads to irreversible damages.

Moreover, patient setup using the proton beam itself would be beneficial for delivery uncertainties. Currently, clinics have use an X-ray machine that might present a slight misplacement in position compared to the therapeutic proton beam. This is usually clinically irrelevant for double scattering proton treatments since the whole field is treated at once, centered on the tumor with added setup margins. On the other hand, this is a more delicate issue for scanned proton beams as it is considerably more difficult to accurately asses the exact beam position.

These results are expendable to any type of ion radiography system, such as carbon ion imaging, due to their similar physical properties. 


\subsection{Moving tumor cases}

\subsubsection{Lung patients}
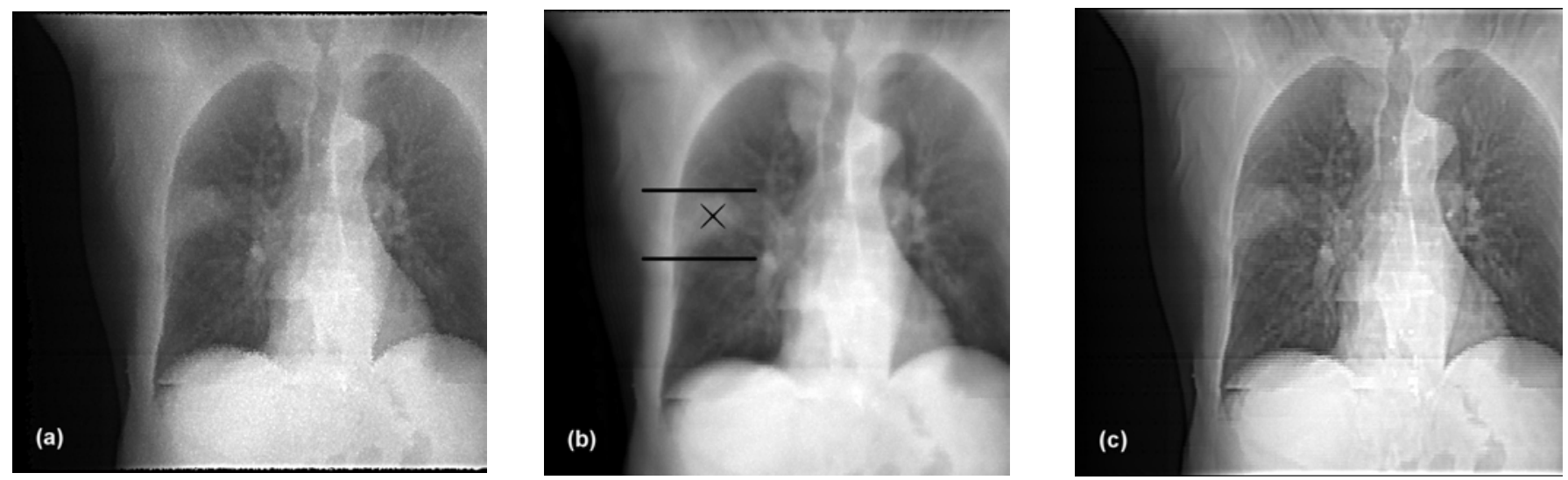

Figure 4: Reconstructed breathing cycles for a lung tumor patients with 1)a 230-MeV proton pencil beam, 2)a 330-MeV proton pencil beam, and 3) a 500-MeV/nucleon carbon ion pencil beam (Please note: these sequences, made in ETTE, must be visualized on a computer using Adobe Acrobat ${ }^{T M}$ in order to properly experience the motion).

Reconstructed radiographs for a lung patient using the three different suggested beams are presented side by side in Figure

For each energy, the tumor edges are defined, and the motion easily appreciable. This shows that, using an ion delivery system for which the particle energy can be quickly modulated, 4D imaging of the patient during treatment is possible. By implementing a "gating" system and synchronizing the imaging process with the beam delivery, this could further lead to direct IGIT.

As expected from earlier studies [3]-[8], a more energetic proton beam (330MeVvs $230 \mathrm{MeV})$ results in better spatial resolution, hence clearer tumor edges and better IGIT capabilities. It also corresponds to a deeper range, hence a longer plateau region of the beam, which results in a significant increase in the maximum patient's thickness that can be imaged.

Figure 5 shows a comparison of juxtaposing 330- $\mathrm{MeV}$ proton radiographs for the two different aforementioned lung patients.

The first/female patient's radiograph (right) presents a slightly decreased spatial comparison versus the second/male patient's image (left). This is mainly due to the difference in body thickness and the presence of a large breast in the female case, which further emphasizes the great potential of using a more energetic beam for imaging purposes.

These two patients have significant differences in tumor location, size and amplitude of motion. The tumor motion is clearly appreciable in both dynamic sequences $(\approx 0.5 \mathrm{~cm}$ in the female case, and $\approx 1 \mathrm{~cm}$ in the male case). This result highlights the fact that radiography could be used for IGIT for a large range of clinically relevant tumor motion amplitudes and locations within the lungs. 

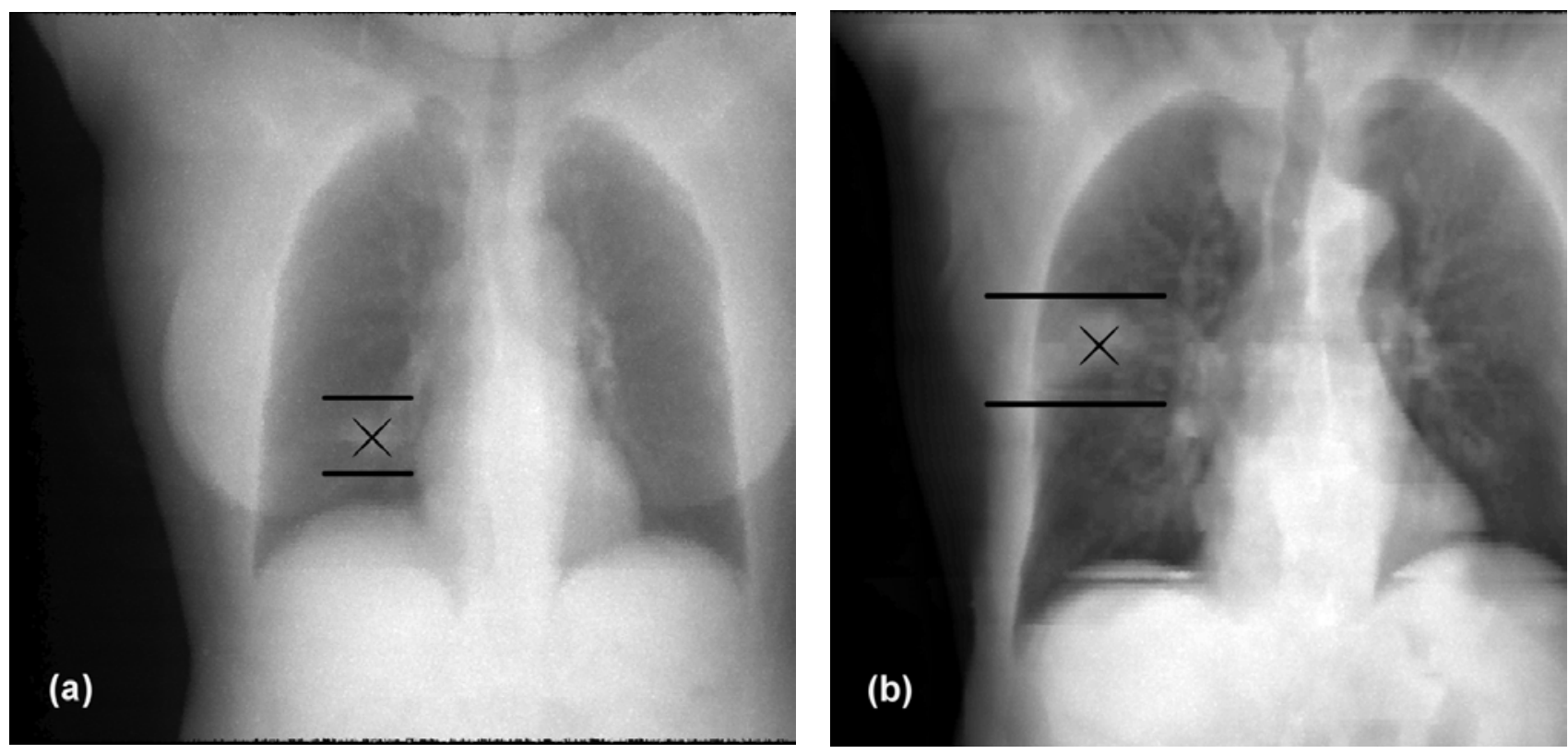

Figure 5: Reconstructed breathing cycles for two lung tumor patients (a)female, b) male) with clear visualization of tumor motion. These were produced from 330-MeV proton pencil beam radiographs. The motions extent is shown between the lines, and the center of the tumor was highlighted with a cross (Please note: these sequences, made in ${ }^{A} T_{E} X$, must be visualized on a computer using Adobe Acrobat ${ }^{T M}$ in order to properly experience the motion).

\subsubsection{Liver patients}

Figure 6 shows reconstructed radiographs for the selected liver tumor patient.
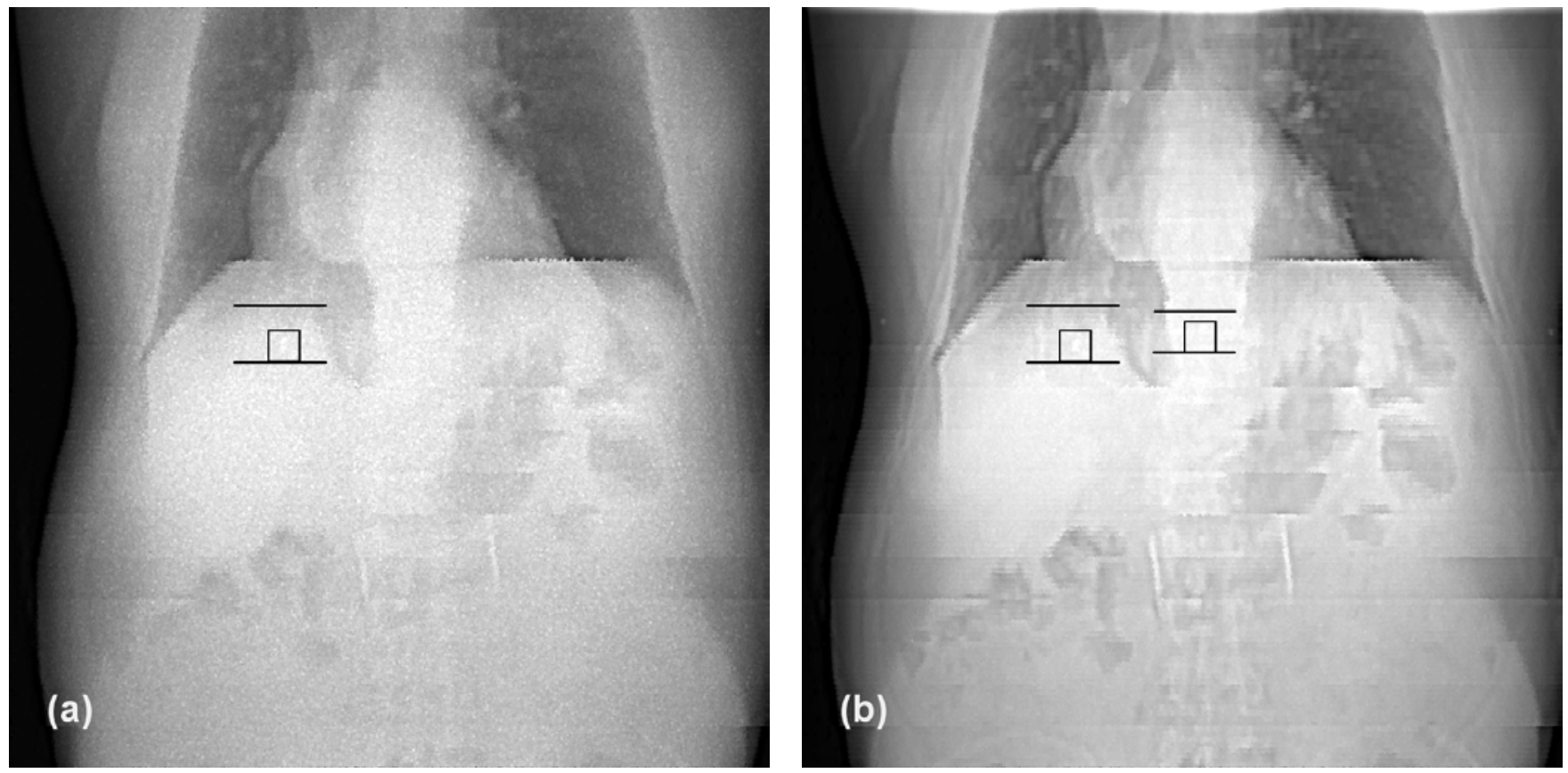

Figure 6: Reconstructed breathing cycles for a liver tumor patient with 1)a 330-MeV proton pencil beam, and 2)a 500-MeV/nucleon carbon ion pencil beam (Please note: these sequences, made in $E^{A} T_{E} X$, must be visualized on a computer using Adobe Acrobat ${ }^{T M}{ }_{i n}$ order to properly experience the motion). The visible fiducial markers inserted prior to radiotherapy are highlighted by the squares on the pictures, and the motion maxima are shown with the horizontal lines. Furthermore, the bright lines at the bottom of the patient correspond to the glass box used for breathing tracking and should not be mistaken for the fiducial markers..

The difficulty of defining the tumor edges in liver cancer patients comes from the homogeneity between the tumor tissue 
and its surrounding healthy tissue; especially from a density point of view ( $\approx 1.04$ for the tumor versus $\approx 1.0$ for soft tissue). Liver cases are therefore considerably more difficult than lung cases from an imaging standpoint. Due to this limitation, it is common to insert gold seeds (fiducial markers) around the tumor for patient set-up purposes. These fiducial markers can therefore be used to locate the tumor in real-time and lead to IGPT. However, not shown here, a 230- $M e V$ pencil proton beam fails to highlight the fiducial markers due to the MCS component of the beam. The latter results in a lower spatial resolution of the images, thus makes a "low" energy beam not suitable for indirect IGIT. On the other hand, the fiducial markers are visible on both a 330- $\mathrm{MeV}$ proton pencil beam, and a $500-\mathrm{MeV} /$ nucleon carbon pencil beam radiographs. Depending of the seeds location (cf seed in spine alignment in Figure 6), one can have difficulties discerning the gold seeds with protons, even at higher energies $(330-\mathrm{MeV})$. Indeed, the seed aligned with the spine is not visible. Indirect IGPT could therefore only be implemented at centers with a high energy beam line ( $\mathrm{ge} 330 \mathrm{MeV})$ and most likely on a patient specific basis. Given their intrinsic higher imaging capabilities, carbon ions leads to a slightly better definition of the fiducial markers' edges, and are thence more suitable for indirect IGIT.

A limitation of this study resides in the use of the CT data set to obtain patient images. Indeed, it was shown that ion radiography offers much greater density resolution than conventional radiography [8], and simulating through an "actual" patient might lead to better results than the ones discussed in this study. Furthermore, with a more advance imaging system such as ion CT, and provided that the tumor edges can be clearly defined for difficult cases such as in liver patients, one might be able to entirely suppress the need for fiducial markers. In such case, indirect IGIT would not be feasible anymore, and direct IGIT might not be suitable either due to the lower resolution of an ion radiograph versus a ion CT.

\section{Conclusion}

Evaluation of ion radiographs generated using the PR-creator software shows that our reconstruction algorithm offers high image quality. This further translates into the clinical relevance of ion radiography which could improve both setup and radiotherapeutic treatment.

The use of ion radiography for patient setup would not only minimize the imaging dose delivered to the patient, especially significant in pediatric cases, but also reduce the delivery uncertainties by using a single

The high spatial resolution of ion radiography also results in easy tumor edges' detection in lung, hence the feasibility of using direct tumor tracking.

In more homogeneous tumor site such as in liver patient, the tumor edges are not clearly visible, and the use of fiducial markers can participate in indirect tumor tracking. The latter however, necessitate a high energy proton beam $(\geq 330 \mathrm{MeV})$ as the limited spatial resolution at lower energies makes it impossible to accurately determine the fiducial markers position. Carbon beams can further help overcome this issue due to their known greater intrinsic imaging properties.

This highlights the possibility of using ion radiography for IGIT purposes with a machine appropriately tuned. The use of IGIT could further lead to adaptive ion therapy in which the dosimetry and tumor position are constantly taken into account during the delivery process.

It should be noted that CT-based simulations are convenient for patient imaging studies, however this process might results in an uncertainty in the body composition. A future study using a better simulation model for the patient could lead to better results than the ones presented in the study.

\section{References}

[1] V. Steward, A. Koehler, "Proton Beam Radiography in Tumor Detection," Science 179, 4076, 913-914 (1973).

[2] K. Hanson et al., "Proton computed tomography of human specimens," Phys. Med. Biol. 27, 25 (1982). 
[3] U. Schneider, E. Pedroni, "Multiple Coulomb Scattering and spatial resolution in proton radiography," Med. Phys. 21, 11, 1657-1663 (1994).

[4] R. Schulte et al., "Density resolution of proton computed tomography," Med. Phys. 32, 4, 1035-1046 (2005).

[5] N. Depauw et al, "Preliminary study of proton radiography imaging qualities using GEANT4 Monte Carlo simulations," Nuclear Technology, 175, 1, 6-10 (2011).

[6] H. Ryu et al., "Density and spatial resolutions of proton radiography using a range modulation technique," Phys. Med. Biol. 53, 19, 5461-5468 (2008).

[7] Li Tianfang et al., "Reconstruction for proton computed tomography by tracing proton trajectories A Monte Carlo study," Med. Phys. 33, 3, 699-706 (2006).

[8] N. Depauw, J. Seco, "Sensitivity study of proton radiography and comparison with kV and MV x-ray imaging using GEANT4 Monte Carlo simulations," Phys. Med. Biol. 56, 2407-2421 (2011).

[9] J. Seco, N. Depauw, "Proof of principle study of the use of a CMOS active pixel sensor for proton radiography," Med. Phys. 38, 2, 622-623 (2011).

[10] S. Agostinelli et al., "Geant4 - a simulation toolkit," Nuclear Instrumentation Methods Phys. Res. A 506, 250-303 (2003).

[11] C. Z. Jarlskog, H. Paganetti, "Physics Settings for Using the Geant4 Toolkit in Proton Therapy," IEEE Trans. Nucl. Sci. 55, 1018-1025 (2008).

[12] H. Paganetti et al, "Clinical implementation of full Monte Carlo dose calculation in proton beam therapy," Phys. Med. Biol. 53, 17, 4825-4853 (2008).

[13] MATLAB 2010b, The MathWorks Inc., Natick, MA, (2000).

[14] Health Physics Society, "Radiation exposure from medical diagnostic imaging procedures," HPS fact sheet (2006).

[15] C. Walter et al., "Phantom and in-vivo measurements of dose exposure by image-guided radiotherapy (IGRT): MV portal images vs. kV portal images vs. cone-beam CT"," Radiother. Oncol. 85, Issue 3, 418-426 (2007). 